\title{
Erratum to: Investigation of Commuting Hamiltonian in Quantum Markov Network
}

\author{
Farzad Ghafari Jouneghani • Mohammad Babazadeh • \\ Rogayeh Bayramzadeh $\cdot$ Hossein Movla
}

Published online: 30 September 2014

(C) Springer Science+Business Media New York 2014

\section{Erratum to: Int J Theor Phys (2014) 53:2521-2530 \\ DOI 10.1007/s10773-014-2042-8}

The above authors wish to correct some typographical errors found in the original article. Eqs. (2) and (7) have writing mistakes. The correct equations are found below:

$$
\begin{gathered}
I(A: C \mid B)=H(A, B)+H(C, B)-H(A, B, C)-H(B) \\
I(A: C \mid B)=S(A, B)+S(C, B)-S(A, B, C)-S(B)
\end{gathered}
$$

At page no 2528 of the original paper, paragraph under Eq. (11e) lines 3 and 4 should be read as "For a sample, we can see Fig. 7 which illustrate the $I(A: C \mid B)$, where $A, C$ and $B$ comprise spins (1\&2), (4\&5) and 3 respectively.

Everything else remains unchanged.

The online version of the original article can be found at http://dx.doi.org/10.1007/s10773-014-2042-8.

F. Ghafari Jouneghani $(\varangle) \cdot$ M. Babazadeh $\cdot$ R. Bayramzadeh Department of Physics, University of Tabriz, Tabriz, Iran

e-mail: f.ghafari89@ms.tabrizu.ac.ir

H. Movla

Azar Aytash Co., Technology Incubator, University of Tabriz, Tabriz, Iran 